\title{
Variational collision integrator for polymer chains
}

\author{
Sigrid Leyendecker ${ }^{\mathrm{a}}$, Carsten Hartmann ${ }^{\mathrm{b}}$, Michael Koch ${ }^{\mathrm{a}}$ \\ ${ }^{a}$ Chair of Applied Dynamics, University of Erlangen-Nuremberg, Germany \\ ${ }^{b}$ Institut für Mathematik, Freie Universität Berlin, Germany
}

\begin{abstract}
The numerical simulation of many-particle systems (e.g., in molecular dynamics) often involves constraints of various forms. We present a symplectic integrator for mechanical systems with holonomic (bilateral) and unilateral contact constraints, the latter being in the form of a nonpenetration condition. The scheme is based on a discrete variant of Hamilton's principle in which both the discrete trajectory and the unknown collision time are varied (cf. [Fetecau et al., 2003, SIAM J. Applied Dynamical Systems, 2, pp. 381-416]). As a consequence, the collision event enters the discrete equations of motion as an unknown that has to be computed on-the-fly whenever a collision is imminent. The additional bilateral constraints are efficiently dealt with employing a discrete null space reduction (including a projection and a local reparametrisation step) which considerably reduces the number of unknowns and improves the condition number during each time-step as compared to a standard treatment with Lagrange multipliers. We illustrate the numerical scheme with a simple example from polymer dynamics, a linear chain of beads, and test it against other standard numerical schemes for collision problems.
\end{abstract}

Keywords: variational integrators, collisions, holonomic constraints, discrete null space method, event-driven algorithm, polymer dynamics, chain of beads

\section{Introduction}

In computer simulations of polymeric solutions, the polymers are often thought of as spherical hard bodies (beads) with no internal degrees of freedom, but covalent and non-bonded interactions between them [11]. The typical interaction potentials (such as the Lennard-Jones potential) are very stiff, which limits the maximum possible time-step in a simulation. A common approach to increase the time-step in order to speed up the simulation consists in replacing the stiff forces by constraints [31]. Pushing this idea to the extreme, one can think of a model in which each molecule is represented as a single bead and the sole interactions are in form of perfectly elastic collisions, modelled by inequality constraints that prevent the beads from penetrating each other (excluded volume effect); this is the basis of the classical event-driven algorithm [1], in which no numerical discretization at all is needed as the motion of the beads follows straight lines in the absence of collisions. For chain-like polymers, a common model is the pearl-necklace-model in which the beads are linked by bond constraints with no other restrictions on their relative positions [29, 14]. It has proven useful for the simulation of melts, and, though conceptually rather simple, is able to explain a variety of phenomena such as reptation, Rouse dynamics, or glassy states on the basis of Monte-Carlo simulations (see [5] and the references therein). Even though the total energy of a chain is independent of the bond angles, within the limitation that the beads 
cannot penetrate each other, it is possible to control the stiffness of the chain (more precisely: its persistence length) by changing the ratio between the bead size and the bond length. The dynamics of the chain are characterised by smooth motions of the bond-constrained particles with impulsive forces when particles collide where the main difficulty for the numerical discretization is the robust treatment of both equality constraints and collisions (see, e.g., [36, 10]). For a recent overview of coarse-grained polymer models, we refer to [28].

In this article we present an variational algorithm that handles equality and inequality constraints on the same footing. Our approach closely follows the route described in [13] where a seamless variational integrator for collision problems is introduced; by 'seamless' we mean that both the integration scheme for the free motion and the contact conditions at the points of collision follow from a single variational principle. Here we extend the seamless variational scheme to the case where, besides the non-penetration condition for the beads, additional holonomic constraints are present during the entire motion. Although our method is an ordinary time-stepping scheme, it shares some of the spirit of event-driven methodology in that it combines an implicit integrator for the free motion of the chain that allows for large time-steps with an exact treatment of the collision events. In particular, the collision times are computed on-the-fly by solving a simple quadratic equation. As the system is not stiff-it does not involve soft-core potentials - the method allows for using relatively large time-steps in the implicit integrator where 'relative' is meant in comparison with a typical molecular dynamics (MD) integrator, e.g., the Verlet method. We stress, however, that it is possible to add an interaction potential; since the scheme that we propose is fully implicit, we do not expect that this will lead to serious time-step limitations or major implementation issues (depending on the implicit solver that is used). But since the main bottleneck for the numerics is in the constraints we focus only on this aspect.

The typical approach for treating hard-core collisions in molecular simulations is by means of combining a traditional MD integrator, typically the Verlet method, with a suitable collision operator that comes into play whenever a collision is detected [32, 27, 33]. Imminent collisions are detected by checking whether particles overlap at the end or during the time interval, while the momentum update at the collision point is done by exploiting the balance of total energy and total linear and angular momentum $[18,7,17]$. The underlying mathematical theory for problems of this type (i.e., non-smooth problems) is provided by the theory of differential inclusions in which the impulsive collision forces are understood as measure-valued generalisations of smooth forces; for a thorough treatment of numerical methods for differential inclusions we refer to [12] or [23]. For smooth systems, variational integrators with constant time-step preserve various properties of the exact dynamics such as symplecticity or momentum maps (e.g., linear or angular momentum) at the discrete level $[34,26]$. The key idea is simple: rather than discretising the continuous-time equations of motion, variational integrators directly discretise Hamilton's principle by appropriate quadrature rules; the numerical scheme then follows from the now discrete Euler-Lagrange equations. Variational integrators have proven useful, e.g., in understanding the long-term stability of symplectic integrators using backward error analysis [16]. Moreover, the (discrete) variational principle allows for easy generalisations so as to treat, e.g., infinite-dimensional systems [8], systems with constraints [19], or contact problems in continuum mechanics [9]. The standard molecular dynamics algorithm for systems with holonomic constraints, that is in fact a variational integrator, is the SHAKE/RATTLE algorithm [31, 2] which can be considered an augmented version of the Verlet algorithm where the constraints are enforced by suitable Lagrange multipliers. As an alternative to Lagrange multipliers that may cause stability problems when the integration time-steps are small (e.g., close to a collision point) the discrete null space method eliminates the constraint force and the Lagrange multipliers by 
projecting the forces in the system onto the space of admissible momenta. It does so by taking advantage of the d'Alembert principle that states that the constraint forces are always acting perpendicular to the constraint manifold and therefore have no component in the tangential direction. The discrete null space method has been introduced in conjunction with an energy-momentum conserving time integration scheme in $[3,4]$ and has been transferred to variational time-stepping schemes in [25]. The new idea in this work is to use the discrete null space methodology also for the treatment of the contact constraints while they are active. As a consequence, the proposed method is variational. Yet the method is not symplectic in any obvious sense (neither are variants based on SHAKE/RATTLE), for the time-stepping is altered in the presence of collisions. Therefore backward error analysis cannot be employed to analyse the long-term stability or quasi-conservation of energy (cf. [7]), even though our numerical test suggest good long-term stability of the scheme.

The paper is organised as follows: Section 2 briefly reviews the variational principle for collision problems. The discrete formulation is given in detail in Section 3 where also the discrete null space method is introduced. As a numerical test of our integrator we simulate a linear chain of beads in Section 4 and compare it against an high-order implicit collocation method and the semi-explicit SHAKE/RATTLE algorithm; the test systems are nontrivial (one is non-integrable), yet simple enough to illustrate the basic features of the method. We summarise the results of the article in Section 5.

\section{Hamilton's principle for collision problems}

Our formulation of the variational collision integrator follows the route taken in [13] and extends it to the case of a system that, besides a non-penetration condition, is subject to holonomic constraints. For a better understanding of the approach, it is instructive to look at the continuous formulation first. Let $Q \subseteq \mathbb{R}^{d}$ denote the $d$-dimensional configuration space of our system where $d$ equals three times the number of particles. We suppose that the system is subject to $m$ holonomic constraints $\boldsymbol{g}(\boldsymbol{q})=\mathbf{0}$ with $\boldsymbol{g}=\left(g_{1}, \ldots, g_{m}\right)^{T}$ being the vector of constraints with the requirement that $\boldsymbol{G}=\nabla \boldsymbol{g}$ has maximum rank $m$ on the admissible set of configurations, $C=\{\boldsymbol{q} \in Q: \boldsymbol{g}(\boldsymbol{q})=\mathbf{0}\} \subset Q$, and with $\operatorname{det} \boldsymbol{G} \boldsymbol{G}^{T} \geq a>0$ being bounded away from zero. ${ }^{1}$ The $m_{c}$ non-penetration conditions for the particles can be expressed in terms of a vector of smooth unilateral constraints $\boldsymbol{g}_{c}(\boldsymbol{q}) \geq \mathbf{0}$ by which the set of admissible configurations turns out to be

$$
C_{+}=\left\{\boldsymbol{q} \in Q: \boldsymbol{g}(\boldsymbol{q})=0, \boldsymbol{g}_{c}(\boldsymbol{q}) \geq \mathbf{0}\right\} \subset C .
$$

Calling

$$
T Q=\left\{(\boldsymbol{q}, \boldsymbol{v}): \boldsymbol{q} \in Q, \boldsymbol{v} \in T_{\boldsymbol{q}} Q\right\}
$$

the state space of the system consisting of the unconstrained positions and velocities, we define the Lagrangian (we use the natural identification of all tangent spaces $T_{q} Q$ with $\mathbb{R}^{d}$ )

$$
L: T Q \rightarrow \mathbb{R}, \quad L(\boldsymbol{q}, \boldsymbol{v})=\frac{1}{2} \boldsymbol{v} \cdot \boldsymbol{M v}-V(\boldsymbol{q})
$$

with $\boldsymbol{M} \in \mathbb{R}^{d \times d}$ being the symmetric and positive definite mass matrix and $V$ being a smooth potential energy. Now let $\gamma:[0, T] \rightarrow Q$ be a curve in $Q$ that is everywhere twice continuously

${ }^{1}$ The latter requires that the function $g$ has a certain regularity that we can safely assume for most cases of interest. 
differentiable except at an isolated impact point $\boldsymbol{q}_{\iota}=\gamma\left(t_{t}\right), t_{\iota} \in(0, T)$ where the curve $\gamma$ hits the boundary $\partial C_{+}$of the admissible set (i.e., where exactly one of the components of $\boldsymbol{g}_{c}$ is zero). The classical action is then of the form

$$
S\left[\gamma, t_{l}\right]=\int_{0}^{t_{\iota}} \hat{L}(\gamma(t), \dot{\gamma}(t)) d t+\int_{t_{\iota}}^{T} \hat{L}(\gamma(t), \dot{\gamma}(t)) d t
$$

where

$$
\hat{L}(\boldsymbol{q}, \boldsymbol{v})=L(\boldsymbol{q}, \boldsymbol{v})-\boldsymbol{g}(\boldsymbol{q}) \cdot \lambda
$$

is the augmented Lagrangian involving the constraints $g$ and the Lagrange multiplier $\lambda \in \mathbb{R}^{m}$ (cf. Lagrange multiplier theorem e.g. in [35]). Taking variations with respect to both $\gamma$ and the unknown collision time $t_{\iota}$, we find

$$
\begin{aligned}
\delta S\left[\gamma, t_{\iota}\right] & =\delta\left(\int_{0}^{t_{\iota}} \hat{L}(\gamma(t), \dot{\gamma}(t)) d t+\int_{t_{\iota}}^{T} \hat{L}(\gamma(t), \dot{\gamma}(t)) d t\right) \\
& =\int_{0}^{T}\left(\frac{\partial \hat{L}}{\partial \gamma}-\frac{d}{d t} \frac{\partial \hat{L}}{\partial \dot{\gamma}}\right) \cdot \delta \gamma d t-\left[\frac{\partial \hat{L}}{\partial \dot{\gamma}} \cdot \delta \gamma+\hat{L} \delta t_{\iota}\right]_{t_{\iota}^{-}}^{t_{\iota^{+}}} .
\end{aligned}
$$

Requiring that the integral vanishes, yields Newton's law

$$
\boldsymbol{M} \ddot{\boldsymbol{q}}=-\nabla V(\boldsymbol{q})-\boldsymbol{G}(\boldsymbol{q})^{T} \lambda, \quad \boldsymbol{g}(\boldsymbol{q})=\mathbf{0}
$$

for the motion away from the collision. The remaining boundary terms provide the contact conditions at the point of impact. To make this precise we call $g_{c}$ the scalar component of $\boldsymbol{g}_{c}$ that is zero at the moment of collision (cf. 3.2 for the treatment of multiple collisions at a time) and note that $g_{c}\left(\gamma\left(t_{l}\right)\right)=0$ entails $\delta g_{c}\left(\gamma\left(t_{l}\right)\right)=0$, i.e.,

$$
G_{c}\left(\gamma\left(t_{\iota}\right)\right) \cdot\left(\delta \boldsymbol{q}_{\iota}+\dot{\gamma}\left(t_{\iota}\right) \delta t_{\iota}\right)=0
$$

where $\boldsymbol{q}_{\iota}=\gamma\left(t_{t}\right)$ denotes the impact point. The last equation is satisfied if either

$$
\delta \boldsymbol{q}_{\iota}=-\dot{\gamma}\left(t_{\iota}\right) \delta t_{\iota}
$$

or

$$
\delta \boldsymbol{q}_{\iota} \perp G_{c}\left(\boldsymbol{q}_{\iota}\right) \text { for } \delta t_{\iota}=0
$$

which determines the admissible variations of the curve at the collision point. Taking joint variations of $\boldsymbol{q}_{\iota}$ and $t_{\iota}$ under the constraint $\delta \boldsymbol{q}_{\iota}=-\dot{\gamma}\left(t_{\iota}\right) \delta t_{\iota}$ implies

$$
\left[\hat{L}-\frac{\partial \hat{L}}{\partial \dot{\gamma}} \cdot \dot{\gamma}\right]_{t_{i}^{-}}^{t_{t}^{+}}=0
$$

which, using that

$$
E=\frac{\partial \hat{L}}{\partial \dot{\gamma}} \cdot \dot{\gamma}-\hat{L},
$$

is equivalent to conservation of energy $E$. Conversely, if we vary $\boldsymbol{q}_{\iota}$ orthogonal to the contact surface while keeping $t_{\iota}$ fixed (i.e., $\delta t_{\iota}=0$ ), then

$$
\left[\frac{\partial \hat{L}}{\partial \dot{\gamma}} \cdot \delta \gamma\right]_{t_{\iota}^{-}}^{t_{t}^{+}}=0 \quad \text { for } \quad \delta \gamma\left(t_{\iota}\right) \perp G_{c}\left(\boldsymbol{q}_{\iota}\right)
$$


implies

$$
\left[\frac{\partial \hat{L}}{\partial \dot{\gamma}}\right]_{t_{i}^{\tau_{i}}}^{t_{i}^{+}}=G_{c}^{T} \lambda_{c} .
$$

Here $\lambda_{c} \in \mathbb{R}$ is an unknown Lagrange multiplier that must be determined by solving (5). Defining the conjugate momentum $\boldsymbol{p}=\partial L / \partial \dot{\gamma}$ in the usual way, equations (5) and (6) can be recast as

$$
\boldsymbol{p}\left(t_{l}^{+}\right)=\boldsymbol{p}\left(t_{\iota}^{-}\right)+G_{c}^{T}\left(\boldsymbol{q}_{\iota}\right) \lambda_{c}, \quad \boldsymbol{p}\left(t_{\iota}^{+}\right) \cdot \boldsymbol{M}^{-1} \boldsymbol{p}\left(t_{\iota}^{+}\right)=\boldsymbol{p}\left(t_{\iota}^{-}\right) \cdot \boldsymbol{M}^{-1} \boldsymbol{p}\left(t_{l}^{-}\right)
$$

But the last equation is simply the momentum reflection law for an elastic collision. That is, the change in momentum occurs normal to the contact surface where the amount by which the momentum changes is determined from the conservation of energy during the collision (note that the potential $V$ is not needed to determine the unknown multiplier $\lambda_{c}$ ). As a consequence of (7), both total linear and angular momentum and the total energy are conserved during the collision.

We stress that the contact condition (7) follows seamlessly from the boundary terms arising in (3). In the next section we will show, again following the approach described in [13], how the discrete variant of (1) naturally gives rise to a fully variational collision integrator.

\section{Variational collision integrator for constrained problems}

Our derivation below follows closely the route taken in [13], but extends it to the case of systems subject to holonomic constraints. The constraints are treated fully variationally using the discrete null space method that has been introduced in [25]. The new idea here is to use the discrete null space methodology also for the treatment of the contact forces while they are active.

\subsection{Discrete variational principle and discrete null space reduction}

Assume that the time nodes $t_{0}, t_{1}, \ldots, t_{l-1}, t_{l+1}, \ldots, t_{N}$ with a constant basic time-step $h=$ $t_{n+1}-t_{n}$ are given, however the collision time $t_{\iota}$ with $t_{t-1} \leq t_{\iota} \leq t_{l+1}$ is unknown. Let the discrete trajectory be denoted by $\boldsymbol{q}_{d}=\left\{\boldsymbol{q}_{n}\right\}_{n=0}^{N}$ with $\boldsymbol{q}_{n} \approx \boldsymbol{q}\left(t_{n}\right)$, and let $\lambda_{d}=\left\{\lambda_{n}\right\}_{n=0}^{N}$ with $\lambda_{n} \approx \lambda\left(t_{n}\right)$ approximate the Lagrange multipliers. As usual in the context of discrete variational principles (e.g., see [26]), the discrete Lagrangian is an approximation to the action integral of the continuous Lagrangian over one time interval. In accordance with (2), this yields away from a collision

$$
\hat{L}_{d}\left(\boldsymbol{q}_{n}, \boldsymbol{q}_{n+1}, t_{n}, t_{n+1}\right) \approx \int_{t_{n}}^{t_{n+1}} L(\boldsymbol{q}, \boldsymbol{v})-\boldsymbol{g}(\boldsymbol{q}) \cdot \lambda d t
$$

In this work, a midpoint discrete Lagrangian

$$
L_{d}\left(\boldsymbol{q}_{n}, \boldsymbol{q}_{n+1}, t_{n}, t_{n+1}\right)=\left(t_{n+1}-t_{n}\right) L\left(\frac{\boldsymbol{q}_{n}+\boldsymbol{q}_{n+1}}{2}, \frac{\boldsymbol{q}_{n+1}-\boldsymbol{q}_{n}}{t_{n+1}-t_{n}}\right)
$$

is used in the following discrete augmented Lagrangian

$$
\hat{L}_{d}\left(\boldsymbol{q}_{n}, \boldsymbol{q}_{n+1}, t_{n}, t_{n+1}\right)=L_{d}\left(\boldsymbol{q}_{n}, \boldsymbol{q}_{n+1}, t_{n}, t_{n+1}\right)-\frac{t_{n+1}-t_{n}}{2}\left(\boldsymbol{g}\left(\boldsymbol{q}_{n}\right) \cdot \lambda_{n}+\boldsymbol{g}\left(\boldsymbol{q}_{n+1}\right) \cdot \lambda_{n+1}\right) .
$$

For the clarity of exposition, the dependence of the discrete Lagrangian on given time nodes is not stated explicitly in the sequel and it is assumed that only one collision $g_{c}\left(\boldsymbol{q}_{t}\right)=0$ occurs at $t_{\iota}$ during the time interval $\left[t_{\iota-1}, t_{l+1}\right]$; see Figure 1 for an illustration. The extension to multiple 


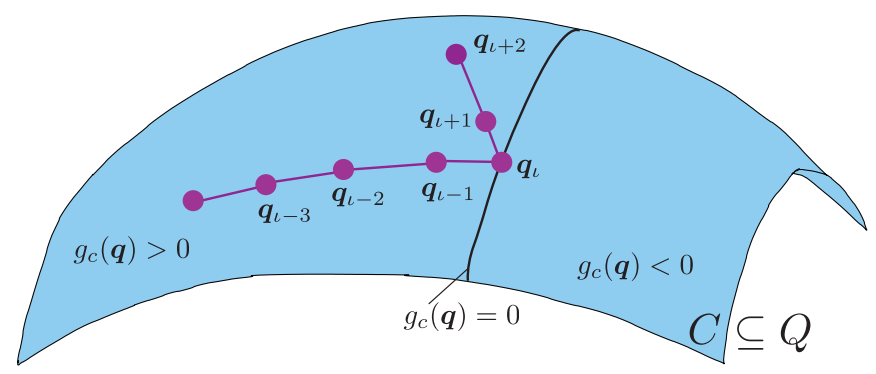

Figure 1: Discrete trajectory in constraint manifold.

collisions is formally straightforward, however more involved from the implementation point of view (see 3.2). An approximation to (1) is given by the discrete action sum

$$
\begin{aligned}
S_{d} & =\sum_{n=0}^{\iota-2} L_{d}\left(\boldsymbol{q}_{n}, \boldsymbol{q}_{n+1}\right)-\frac{t_{n+1}-t_{n}}{2}\left(\boldsymbol{g}\left(\boldsymbol{q}_{n}\right) \cdot \lambda_{n}+\boldsymbol{g}\left(\boldsymbol{q}_{n+1}\right) \cdot \lambda_{n+1}\right) \\
& +L_{d}\left(\boldsymbol{q}_{\iota-1}, \boldsymbol{q}_{\iota}, t_{\iota-1}, t_{\iota}\right)-\frac{t_{\iota}-t_{\iota-1}}{2}\left(\boldsymbol{g}\left(\boldsymbol{q}_{\iota-1}\right) \cdot \lambda_{\iota-1}+\boldsymbol{g}\left(\boldsymbol{q}_{\iota}\right) \cdot \lambda_{\iota}+g_{c}\left(\boldsymbol{q}_{\iota}\right) \cdot \lambda_{c}\right) \\
& +L_{d}\left(\boldsymbol{q}_{\iota}, \boldsymbol{q}_{\iota+1}, t_{\iota}, t_{\iota+1}\right)-\frac{t_{\iota+1}-t_{\iota}}{2}\left(\boldsymbol{g}\left(\boldsymbol{q}_{\iota}\right) \cdot \lambda_{\iota}+\boldsymbol{g}\left(\boldsymbol{q}_{\iota+1}\right) \cdot \lambda_{\iota+1}+g_{c}\left(\boldsymbol{q}_{\iota}\right) \cdot \lambda_{c}\right) \\
& +\sum_{n=\iota+1}^{N-1} L_{d}\left(\boldsymbol{q}_{n}, \boldsymbol{q}_{n+1}\right)-\frac{t_{n+1}-t_{n}}{2}\left(\boldsymbol{g}\left(\boldsymbol{q}_{n}\right) \cdot \lambda_{n}+\boldsymbol{g}\left(\boldsymbol{q}_{n+1}\right) \cdot \lambda_{n+1}\right) .
\end{aligned}
$$

As before, the discrete variational principle for the constrained motion requires that $\delta S_{d}=0$ for all admissible variations $\delta \boldsymbol{q}_{1}, \ldots, \delta \boldsymbol{q}_{\iota}, \ldots, \delta \boldsymbol{q}_{N-1}, \delta \lambda_{1}, \ldots, \delta \lambda_{N}, \delta \lambda_{c}, \delta t_{\iota}$. This then yields discrete equations of motion for the dynamics off the contact surface with additional boundary conditions at the collision points. The equations for the collision-free motion are stated first:

Pre- and post-collision. As long as no collision takes place, i.e., for $n=1, \ldots, \iota-2$ the discrete variational principle yields the following system

$$
\begin{aligned}
D_{2} L_{d}\left(\boldsymbol{q}_{n-1}, \boldsymbol{q}_{n}\right)+D_{1} L_{d}\left(\boldsymbol{q}_{n}, \boldsymbol{q}_{n+1}\right)-\frac{t_{n+1}-t_{n-1}}{2} \boldsymbol{G}^{T}\left(\boldsymbol{q}_{n}\right) \lambda_{n} & =\mathbf{0} \\
\boldsymbol{g}\left(\boldsymbol{q}_{n+1}\right) & =\mathbf{0}
\end{aligned}
$$

which is solved for $\boldsymbol{q}_{2}, \ldots, \boldsymbol{q}_{\iota-1}, \lambda_{1}, \ldots, \lambda_{\iota-2}$. Here, $D_{i} L_{d}$ denotes the derivative of the discrete Lagrangian with respect to the $i$-th argument. Note that just as in the continuous case described in Section 2, there exists a Lagrange multiplier theorem relating stationary points of the discrete action (9) to the finite dimensional system (10), see [26]. Once the collision configuration $\boldsymbol{q}_{\iota}$ and time $t_{\iota}$ and the first post-collision configuration $\boldsymbol{q}_{\iota+1}$ have been determined (as will be described below), normal time-stepping continues for $n=\imath+1, \ldots, N-1$, i.e., (10) is solved for $\boldsymbol{q}_{\iota+2}, \ldots, \boldsymbol{q}_{N}, \lambda_{\iota+1}, \ldots, \lambda_{N-1}$. Obviously, (10) is a two-step method, thus special care must be given to the initialisation of the simulation. Assuming that no collision takes place during the first 
time-step, the following equations determine $\boldsymbol{q}_{1}$ and $\lambda_{0}$ from given initial data $(\boldsymbol{q}(0), \dot{\boldsymbol{q}}(0)) \in T C$. First, one sets $\boldsymbol{q}_{0}=\boldsymbol{q}(0)$ and $\boldsymbol{p}_{0}^{-}=(\partial L / \partial \dot{\boldsymbol{q}})(\boldsymbol{q}(0), \dot{\boldsymbol{q}}(0))$ and then solves

$$
\begin{aligned}
\boldsymbol{p}_{0}^{-}+D_{1} L_{d}\left(\boldsymbol{q}_{0}, \boldsymbol{q}_{1}\right)-\frac{t_{1}-t_{0}}{2} \boldsymbol{G}^{T}\left(\boldsymbol{q}_{0}\right) \lambda_{0} & =\mathbf{0} \\
\boldsymbol{g}\left(\boldsymbol{q}_{1}\right) & =\mathbf{0}
\end{aligned}
$$

Collisions in the first time-step can be handled analogously to later collisions described below.

Remark 1 (SHAKE/RATTLE). If, instead of the midpoint discrete Lagrangian in (8), a constant time-step $h>0$ and any composition

$$
L_{d}\left(\boldsymbol{q}_{n}, \boldsymbol{q}_{n+1}\right)=\alpha h L\left(\boldsymbol{q}_{n}, \frac{\boldsymbol{q}_{n+1}-\boldsymbol{q}_{n}}{h}\right)+(1-\alpha) h L\left(\boldsymbol{q}_{n+1}, \frac{\boldsymbol{q}_{n+1}-\boldsymbol{q}_{n}}{h}\right)
$$

with $0 \leq \alpha \leq 1$ is used as discrete Lagrangian, (10) yields the SHAKE algorithm. Its phase space variant RATTLE that is due to [2] is well known to be symplectic on the constrained phase space $T^{*} C($ e.g., see $[21])$.

Discrete null space reduction. Details on the reduction of the discrete variational equations of motion with constraints via the discrete null space method with local reparametrisation can be found in [25]. The main idea is the elimination of the constraint forces from the discrete system via the premultiplication with an appropriate null space matrix $\boldsymbol{P}(\cdot): \mathbb{R}^{d-m} \rightarrow T C$, i.e., the null space matrix fulfils range $\boldsymbol{P}\left(\boldsymbol{q}_{n}\right)=\operatorname{ker} \boldsymbol{G}\left(\boldsymbol{q}_{n}\right)$. Secondly, a local reparametrisation of the constraint manifold $\boldsymbol{q}_{n+1}=\boldsymbol{F}_{d}\left(\boldsymbol{u}_{n+1}, \boldsymbol{q}_{n}\right) \in C$ in terms of the discrete generalised coordinates $\boldsymbol{u}_{n+1} \in \mathbb{R}^{d-m}$ representing the system's change during one time-step ensures that the constraints are fulfilled and (10b) becomes superfluous. The reduced equations read

$$
\boldsymbol{P}^{T}\left(\boldsymbol{q}_{n}\right)\left[D_{2} L_{d}\left(\boldsymbol{q}_{n-1}, \boldsymbol{q}_{n}\right)+D_{1} L_{d}\left(\boldsymbol{q}_{n}, \boldsymbol{F}_{d}\left(\boldsymbol{u}_{n+1}, \boldsymbol{q}_{n}\right)\right)\right]=\mathbf{0}
$$

Away from the collision, they are solved for $\boldsymbol{u}_{2}, \ldots, \boldsymbol{u}_{t-1}$ and $\boldsymbol{u}_{t+2}, \ldots, \boldsymbol{u}_{N}$ while in the very first step,

$$
\boldsymbol{P}^{T}\left(\boldsymbol{q}_{0}\right)\left[\boldsymbol{p}_{0}^{-}+D_{1} L_{d}\left(\boldsymbol{q}_{0}, \boldsymbol{F}_{d}\left(\boldsymbol{u}_{1}, \boldsymbol{q}_{0}\right)\right)\right]=\mathbf{0}
$$

is solved for $\boldsymbol{u}_{1}$. In contrast to an absolute parametrisation in generalised coordinates with respect to the initial configuration $\boldsymbol{q}_{0}$ reading $\boldsymbol{q}_{n+1}=\boldsymbol{F}_{d}\left(\boldsymbol{u}_{n+1}, \boldsymbol{q}_{0}\right)$, locality of the discrete parametrisation avoids singularities present, e.g., when dealing with large rotations. The described procedure reduces the $(d+m)$-dimensional system (10) to the $(d-m)$-dimensional system (11). Depending on the particular problem under consideration, this can reduce the computational costs substantially. Due to the elimination of the Lagrange multipliers from the set of unknowns, the well known condition problem associated with discretisations of index 3 DAEs is removed. While the condition number of the Jacobian matrix in the linearisation of (10) is of the order $h^{-3}$, the corresponding condition number in (11) is independent of the time-step (see [25]). Note that after solving (11), the Lagrange multipliers can always be determined as a post-processing step if one is interested in the constraint forces. This is particularly important when the contact forces themselves are eliminated using the discrete null space reduction (cf. equation (16) below).

Remark 2 (Iterative solution procedure). Solving the nonlinear system (11) iteratively, e.g., using a Newton-Raphson method, an iterative update procedure $\boldsymbol{q}_{n+1}^{l+1}=\boldsymbol{F}_{d}\left(\boldsymbol{u}_{n+1}^{l+1}, \boldsymbol{q}_{n+1}^{l}\right)$ suggests itself (the upper index l denotes the l-th Newton iteration). Then, simply, $\boldsymbol{P}\left(\boldsymbol{q}_{n+1}^{l^{+1}}\right)$ can be used as 
an approximation of the Jacobian of the discrete reparametrisation $\boldsymbol{F}_{d}$ close to the identity by which the Jacobian matrix that occurs in the linearisation of (11) takes the form

$$
\boldsymbol{P}^{T}\left(\boldsymbol{q}_{n}\right) D_{12} L_{d}\left(\boldsymbol{q}_{n}, \boldsymbol{q}_{n+1}^{l}\right) \boldsymbol{P}\left(\boldsymbol{q}_{n+1}^{l}\right)
$$

The latter can be easily computed analytically and only needs to be evaluated at the respective configurations. Note, however, that the approximant thus defined is not sparse.

Collision. Before integrating forward in a new time-step, the contact inequality condition is checked. If it is violated, i.e., if $g_{c}\left(\boldsymbol{q}_{\iota}\right)<0$, then $\boldsymbol{q}_{\iota}$ is discarded and the collision configuration $\boldsymbol{q}_{\iota}$ and time $t_{\iota}$ as well as $\lambda_{\iota-1}$ are determined by solving

$$
\begin{aligned}
D_{2} L_{d}\left(\boldsymbol{q}_{\iota-2}, \boldsymbol{q}_{\iota-1}\right)+D_{1} L_{d}\left(\boldsymbol{q}_{\iota-1}, \boldsymbol{q}_{\iota}, t_{t-1}, t_{\iota}\right)-\frac{t_{\iota}-t_{t-2}}{2} \boldsymbol{G}^{T}\left(\boldsymbol{q}_{\iota-1}\right) \lambda_{\iota-1} & =\mathbf{0} \\
\boldsymbol{g}\left(\boldsymbol{q}_{\iota}\right) & =\mathbf{0} \\
g_{c}\left(\boldsymbol{q}_{\iota}\right) & =0
\end{aligned}
$$

which have been obtained from $\delta S_{d}=0$ in (9) for variations $\delta \boldsymbol{q}_{\iota-1}, \delta \lambda_{\iota}, \delta \lambda_{c}$. After that, taking admissible variations $\delta \boldsymbol{q}_{\iota}, \delta \boldsymbol{\lambda}_{\iota+1}, \delta t_{\iota}$ yields

$$
\begin{array}{r}
D_{2} L_{d}\left(\boldsymbol{q}_{\iota-1}, \boldsymbol{q}_{\iota}, t_{l-1}, t_{\iota}\right)+D_{1} L_{d}\left(\boldsymbol{q}_{\iota}, \boldsymbol{q}_{\iota+1}, t_{\iota}, t_{l+1}\right)-\frac{t_{\iota+1}-t_{\iota-1}}{2}\left(\boldsymbol{G}^{T}\left(\boldsymbol{q}_{\iota}\right) \lambda_{\iota}+\boldsymbol{G}_{c}^{T}\left(\boldsymbol{q}_{\iota}\right) \lambda_{c}\right)=\mathbf{0} \\
\boldsymbol{g}\left(\boldsymbol{q}_{\iota+1}\right)=\mathbf{0} \\
D_{4} L_{d}\left(\boldsymbol{q}_{\iota-1}, \boldsymbol{q}_{\iota}, t_{t-1}, t_{\iota}\right)+D_{3} L_{d}\left(\boldsymbol{q}_{\iota}, \boldsymbol{q}_{t+1}, t_{\iota}, t_{l+1}\right)=0
\end{array}
$$

from which $\boldsymbol{q}_{\iota+1}, \lambda_{\iota}, \lambda_{c}$ follow. Note that (14c) is resulting from the variation with respect to the time node $t_{\iota}$, thus it is a conservation condition for the discrete energy.

Discrete null space reduction. Equivalent to (13), the reduced system yielding $\boldsymbol{u}_{\iota}, t_{\iota}$ reads

$$
\begin{aligned}
\boldsymbol{P}^{T}\left(\boldsymbol{q}_{\iota-1}\right)\left[D_{2} L_{d}\left(\boldsymbol{q}_{\iota-2}, \boldsymbol{q}_{\iota-1}\right)+D_{1} L_{d}\left(\boldsymbol{q}_{\iota-1}, \boldsymbol{q}_{\iota}, t_{t-1}, t_{\iota}\right)\right] & =\mathbf{0} \\
g_{c}\left(\boldsymbol{q}_{\iota}\right) & =0
\end{aligned}
$$

and instead of solving (14), the unknown $\boldsymbol{u}_{t+1}$ can be obtained from

$$
\begin{array}{r}
\boldsymbol{P}_{c}^{T}\left(\boldsymbol{q}_{\iota}\right) \boldsymbol{P}^{T}\left(\boldsymbol{q}_{\iota}\right)\left[D_{2} L_{d}\left(\boldsymbol{q}_{\iota-1}, \boldsymbol{q}_{\iota}, t_{l-1}, t_{\iota}\right)+D_{1} L_{d}\left(\boldsymbol{q}_{\iota}, \boldsymbol{q}_{l+1}, t_{\iota}, t_{l+1}\right)\right]=\mathbf{0} \\
D_{4} L_{d}\left(\boldsymbol{q}_{\iota-1}, \boldsymbol{q}_{\iota}, t_{l-1}, t_{\iota}\right)+D_{3} L_{d}\left(\boldsymbol{q}_{\iota}, \boldsymbol{q}_{\iota+1}, t_{\iota}, t_{l+1}\right)=0
\end{array}
$$

As mentioned before, the Lagrange multipliers and in particular the contact forces $\boldsymbol{G}_{c}^{T}\left(\boldsymbol{q}_{\iota}\right) \lambda_{c}$ can be computed as a post-processing step.

Symplecticity of the discrete flow. One possibility to check whether the method thus defined is symplectic would be to check whether it belongs to the class of variational Specialized Partitioned Additive Runge-Kutta (variational SPARK) methods for which symplecticity has been proved [20, Thm. 4.1]. However the conditions on the coefficients guaranteeing that the method is symplectic are difficult to check in our case; in particular the role of the variable collision time-step is unclear. Away from the collision, a direct proof consists in eliminating the constraints by using generalised coordinates or, if these are not available, appropriate local coordinates. This route has been taken in [34, Thm. 1] and shows that the two formulations are 
equivalent, when the midpoint rule is employed. Hence for the midpoint rule, the symplecticity of the unconstrained midpoint variational integrator entails the symplecticity of the Lagrange multiplier version. The proof essentially consists in showing that, given a (local) embedding $\phi: \mathbb{R}^{d-m} \rightarrow C \subset \mathbb{R}^{d}, \boldsymbol{u} \mapsto \phi(\boldsymbol{u})$ of the constraint submanifold $C$ into the ambient space $\mathbb{R}^{d}$, the discrete Euler-Lagrange equations for the generalised (local) coordinates $\boldsymbol{u}_{n+1}$ have a solution if and only if the respective equations for the ambient space coordinates $\boldsymbol{q}_{n+1}$ plus the Lagrange multiplier $\lambda_{n+1}$ have a solution satisfying $\boldsymbol{q}_{n+1}=\phi\left(\boldsymbol{u}_{n+1}\right)$. Even though the treatment of the constraints in the discrete Lagrangian (8) is slightly different than in [34], the proof carries over as the discrete Euler-Lagrange equations are the same. As a consequence, the corresponding discrete Lagrangian symplectic form is preserved (cf. [13, Sec. 3.3]); the property is trivially inherited by the discrete nullspace reduction method. Note, however, that the discrete flow $\left(\boldsymbol{q}_{n}, \boldsymbol{p}_{n}\right) \mapsto\left(\boldsymbol{q}_{n+1}, \boldsymbol{p}_{n+1}\right)$ is not a symplectic phase space flow $T^{*} C \rightarrow T^{*} C$, for the simple reason that the discrete momenta $\boldsymbol{p}_{n+1}$ do not satisfy the hidden constraint, in other words: $\boldsymbol{p}_{n+1} \notin T_{\boldsymbol{q}_{n+1}}^{*} C$.

Remark 3 (Backward error analysis). The excellent energy behaviour of variational integrators based on constant time-steps can be explained using backward error analysis [26, 30, 16]. However, in the context of collisions, the systems (13)-(14) or (15)-(16) constitute variational time-stepping equations with a variable time-step. While the discrete trajectory is close to the exact solution of a single modified Hamiltonian system away from collisions, the stability argument implied by backward error analysis is no longer applicable here. In [6], a modified collision Verlet algorithm is proposed that conserves the modified Hamiltonian (to fourth order accuracy using a higher order method) during the collision steps and thereby gains numerical stability. Note that collision times are determined solving a quartic equation in [6]. In the presence of a polynomial potential of degree at most 2, the time-stepping equations proposed in this work are at most quadratic also during the collision steps. We leave the stability analysis of the scheme for future work and simply note here, that the numerical example shown in Section 4.4 illustrates excellent energy behaviour for a simulation with 10000 collisions.

\subsection{Multiple collisions}

If multiple collisions occur at the same time $t_{\iota}$, i.e. $\boldsymbol{g}_{c}(\boldsymbol{q}) \geq \mathbf{0} \in \mathbb{R}^{m_{c}}$ is really vector valued with $m_{c} \in \mathbb{R}$, then $\left(\sum_{k=1}^{m_{c}} g_{c_{k}}\left(\boldsymbol{q}_{\imath}\right)\right) \lambda_{c}$ is used in the discrete action (9). Note that corresponding to the single unknown collision time $t_{\iota}$, the Lagrange multiplier $\lambda_{c}$ is scalar. Variation with respect to $\lambda_{c}$ yields $\left(\sum_{k=1}^{m_{c}} g_{c_{k}}\left(\boldsymbol{q}_{t}\right)\right)=0$ in (13c) which, together with the positiveness of all components, is equivalent to $g_{c_{k}}\left(\boldsymbol{q}_{\iota}\right)=0$ for $k=1, \ldots, m_{c}$. Accordingly, $\left(\sum_{k=1}^{m_{c}} \boldsymbol{G}_{c_{k}}^{T}\left(\boldsymbol{q}_{\iota}\right)\right) \lambda_{c}$ appears in (14a) and then, as for single collisions, the discrete energy conservation condition for the complete system (14c) determines $\lambda_{c}$. The discrete null space reduction works analogously to the single collision case.

If collisions follow each other in quick succession, the algorithm does not return to the regular grid immediately but computes another collision time node within a basic time-step $h$.

\section{Kinematic descriptions and numerical examples}

Before we come to the simulation of a colliding polymer chain, we have to recall that the (discrete) null space method requires that a local parametrisation of the constraint submanifold $C \subset Q$ is available. Hence we address the parametrisation issue first. 


\subsection{Kinematics of a rigid body}

For models that describe a polymer as a chain of rigid bodies (here: beads), local coordinates are the centre of mass and the orientation of each particle. Here we will use a particular parametrisation of the rigid body in terms of the 12-dimensional redundant configuration variable $\boldsymbol{q}=\left(\boldsymbol{\varphi}, \boldsymbol{d}_{1}, \boldsymbol{d}_{2}, \boldsymbol{d}_{3}\right)$ as is described in detail in [4, 25]. The parametrisation uses the centre of mass $\varphi \in \mathbb{R}^{3}$ and the directors $\boldsymbol{d}_{I} \in \mathbb{R}^{3}, I=1,2,3$ representing the orientation of the body. Each director triad is constrained to stay orthonormal during the motion. The corresponding null space matrix and nodal reparametrisation take the form

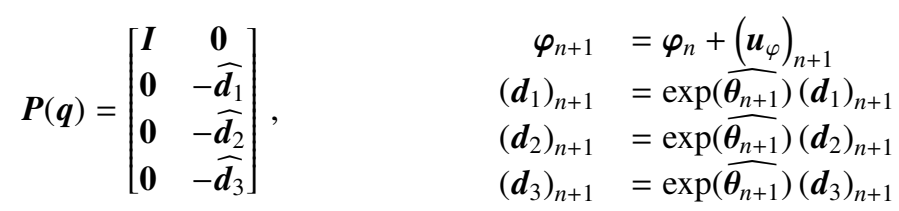

where $\boldsymbol{u}_{n+1}=\left(\left(\boldsymbol{u}_{\varphi}\right)_{n+1}, \boldsymbol{\theta}_{n+1}\right)$ consists of the displacement $\left(\boldsymbol{u}_{\varphi}\right)_{n+1}$ and the incremental rotation vector $\boldsymbol{\theta}_{n+1}$

\subsection{Numerical example: intracolliding rigid spheres}

As a first numerical example, we consider two (oriented) rigid spheres of radius $r=0.1$ with a uniform density $\varrho=2700$ and use a basic time-step of $h=0.01 .^{2}$ The unilateral contact constraint takes the form given in (18) below. The bodies are spinning with angular velocities $\omega_{1}=(1,2,-1)$ and $\omega_{2}=(2,3,-3)$, respectively. Since no forces in tangential direction are exchanged during the collision, the angular velocities remain unchanged during all simulations. In this example, the discrete equations of motion are solved using standard Newton-Raphson iteration (tolerance $10^{-10}$ ) without any damping. In the first simulation, the initial positions and translational velocities of the spheres' centres are $\varphi^{1}=(5,5,0), \dot{\varphi}^{1}=(0,1,0)$ and $\varphi^{2}=$ $(5,7,0), \dot{\varphi}^{2}=(0,-1,0)$. The plots in the first row in Figure 2 show the trajectory of the spheres' centres (whereby their orientation is illustrated by the director triads and the first, contact and last configuration are plotted) as well as the conservation of energy and angular momentum and the evolution of the contact constraint. More precisely, oscillation amplitudes are of the order of magnitude $10^{-13}$ for the energy and $10^{-14}$ for the angular momentum, i.e. oscillations are smaller than the tolerance in the Newton-Raphson iteration, thus energy and angular momentum are considered to be conserved within the numerical tolerance. The middle row shows the results for the initial conditions $\varphi^{1}=(5,5,-0.85), \dot{\varphi}^{1}=(0,1,1)$ and $\varphi^{2}=(5,7,1), \dot{\varphi}^{2}=(0,-1,-1)$. Due to the more complex motion, the energy shows a jump of $9 \cdot 10^{-5}$ at the collision (this jump decreases to $10^{-7}$ for $h=0.001$ and is about $4 \cdot 10^{-3}$ for $\left.h=0.05\right)$, nevertheless, oscillations are small away from the collision (order of magnitude $10^{-13}$ ) and angular momentum is exactly conserved (order of magnitude $10^{-14}$ ) during the entire simulation. In the first simulation, the contact constraint is zero at a given time node and then 6 iterations are necessary to solve (16). In the second simulation, contact occurs between given time nodes, thus (15)-(16) are solved requiring 5 and 13 iterations, respectively. Finally, we consider the initial conditions $\varphi^{1}=(5,5,0.2), \dot{\varphi}^{1}=$ $(0,1,0)$ and $\varphi^{2}=(5,7,0), \dot{\varphi}^{2}=(0,-1,0)$ for which the bodies merely touch each other in passing at a given time node. This case, in which the contact force is very small (depending on the

\footnotetext{
${ }^{2}$ Throughout the article we employ dimensionless units.
} 
tolerance used for collision detection), is sometimes called a 'grazing collision' (cf. [7]). Even though 64 iterations are required here to solve (16), the algorithm finds a solution and proceeds further in time as depicted in the last row in Figure 2. Oscillation amplitudes are of the order of magnitude $10^{-11}$ for the energy of $10^{-14}$ for the angular momentum.

\subsection{Kinematics of a chain of beads}

Consider a chain of $n_{m p}$ mass points illustrated in Figure 3 with mass $m_{i}$ and position $\boldsymbol{q}^{i} \in \mathbb{R}^{3}$, $i=1, \ldots, n_{m p}$, being connected by bond constraints, i.e., the distance between adjacent mass points is constrained to be constant via the $m$-dimensional constraint function $\boldsymbol{g}(\boldsymbol{q})=\mathbf{0}$ reading

$$
g^{i}(\boldsymbol{q})=\frac{1}{2}\left(\left\|\boldsymbol{q}^{i+1}-\boldsymbol{q}^{i}\right\|^{2}-\left(l^{i+1}\right)^{2}\right)=0 \quad \text { for } i=1, \ldots, m
$$

where $m=n_{m p}-1$ and $l^{i+1}$ denotes the initial distance between the mass points $\boldsymbol{q}^{i}$ and $\boldsymbol{q}^{i+1}$. Each mass point is modelled as an (orientationless) sphere of radius $r^{i} \in \mathbb{R}$. The spheres are not allowed to intersect, thus the motion is subject to the inequality constraint

$$
g_{c}^{i j}(\boldsymbol{q})=\frac{1}{2}\left(\left\|\boldsymbol{q}^{i}-\boldsymbol{q}^{j}\right\|^{2}-\left(r^{i}+r^{j}\right)^{2}\right) \geq 0
$$

for all $i \neq j$. In particular, two particles with indices $i$ and $j$ are in contact when $g_{c}^{i j}=0$.

Since the chain is free to move in space, one mass point, say the first one, has three translational degrees of freedom, hence $\dot{\boldsymbol{q}}^{1} \in \mathbb{R}^{3}$, while all others can merely rotate around their neighbours. This gives rise to the following relation of the velocities

$$
\dot{\boldsymbol{q}}^{i}=\dot{\boldsymbol{q}}^{i-1}+\dot{\theta}_{r}^{i} \boldsymbol{r}^{i}+\dot{\theta}_{s}^{i} \boldsymbol{s}^{i} \quad \text { for } i=2, \ldots, n_{m p}
$$

whereby the vectors $\boldsymbol{d}^{i}=\left(\boldsymbol{q}^{i}-\boldsymbol{q}^{i-1}\right) / l^{i}$ and $\boldsymbol{r}^{i}, \boldsymbol{s}^{i}$ form an orthonormal triad. In particular, $\boldsymbol{r}^{i}, \boldsymbol{s}^{i}$ span the tangent plane $T_{d^{i}} S^{2}$. Admissible velocities fulfilling the hidden constraints $\boldsymbol{G}(\boldsymbol{q}) \dot{\boldsymbol{q}}=\mathbf{0}$ can be computed as $\dot{\boldsymbol{q}}=\boldsymbol{P}(\boldsymbol{q}) \boldsymbol{v}$ in terms of the independent velocities $\boldsymbol{v}=\left(\dot{\boldsymbol{q}}^{1}, \dot{\theta}_{r}^{2}, \dot{\theta}_{s}^{2}, \ldots, \dot{\theta}_{r}^{n_{m p}}, \dot{\theta}_{s}^{n_{m p}}\right)$ and the null space matrix

$$
\boldsymbol{P}(\boldsymbol{q})=\left[\begin{array}{cccccccc}
\boldsymbol{I} & \mathbf{0} & \mathbf{0} & \mathbf{0} & \mathbf{0} & \ldots & \mathbf{0} & \mathbf{0} \\
\boldsymbol{I} & \boldsymbol{r}^{2} & \boldsymbol{s}^{2} & \mathbf{0} & \mathbf{0} & \ldots & \mathbf{0} & \mathbf{0} \\
\boldsymbol{I} & \boldsymbol{r}^{2} & \boldsymbol{s}^{2} & \boldsymbol{r}^{3} & \boldsymbol{s}^{3} & \ldots & \mathbf{0} & \mathbf{0} \\
\vdots & \vdots & \vdots & \vdots & \vdots & \ddots & \mathbf{0} & \mathbf{0} \\
\boldsymbol{I} & \boldsymbol{r}^{2} & \boldsymbol{s}^{2} & \boldsymbol{r}^{3} & \boldsymbol{s}^{3} & \ldots & \boldsymbol{r}^{n_{m p}} & \boldsymbol{s}^{n_{m p}}
\end{array}\right]
$$

In particular, $\boldsymbol{P}^{T} \boldsymbol{G}^{T}=\mathbf{0}$ holds and the null space matrix can be used to eliminate the constraint forces from the equations of motion. A null space matrix $\boldsymbol{P}_{c}$ fulfilling $\boldsymbol{P}_{c}^{T} \boldsymbol{P}^{T} \boldsymbol{G}_{c}^{T}=\mathbf{0}$ can easily be defined, e.g., in the case $n_{m p}=3$, it reads

$$
\boldsymbol{P}_{c}(\boldsymbol{q})=\left[\begin{array}{cccc}
\boldsymbol{I} & \mathbf{0} & \mathbf{0} & \mathbf{0} \\
\mathbf{0} & -\left(\boldsymbol{q}^{3}-\boldsymbol{q}^{1}\right) \cdot\left(\boldsymbol{s}^{2}\right) & \mathbf{0} & -\left(\boldsymbol{q}^{3}-\boldsymbol{q}^{1}\right) \cdot\left(s^{3}\right) \\
\mathbf{0} & \left(\boldsymbol{q}^{3}-\boldsymbol{q}^{1}\right) \cdot\left(\boldsymbol{r}^{2}\right) & \mathbf{0} & \mathbf{0} \\
\mathbf{0} & \mathbf{0} & -\left(\boldsymbol{q}^{3}-\boldsymbol{q}^{1}\right) \cdot\left(\boldsymbol{s}^{3}\right) & \mathbf{0} \\
\mathbf{0} & \mathbf{0} & \mathbf{0} & \left(\boldsymbol{q}^{3}-\boldsymbol{q}^{1}\right) \cdot\left(\boldsymbol{r}^{3}\right)
\end{array}\right]
$$



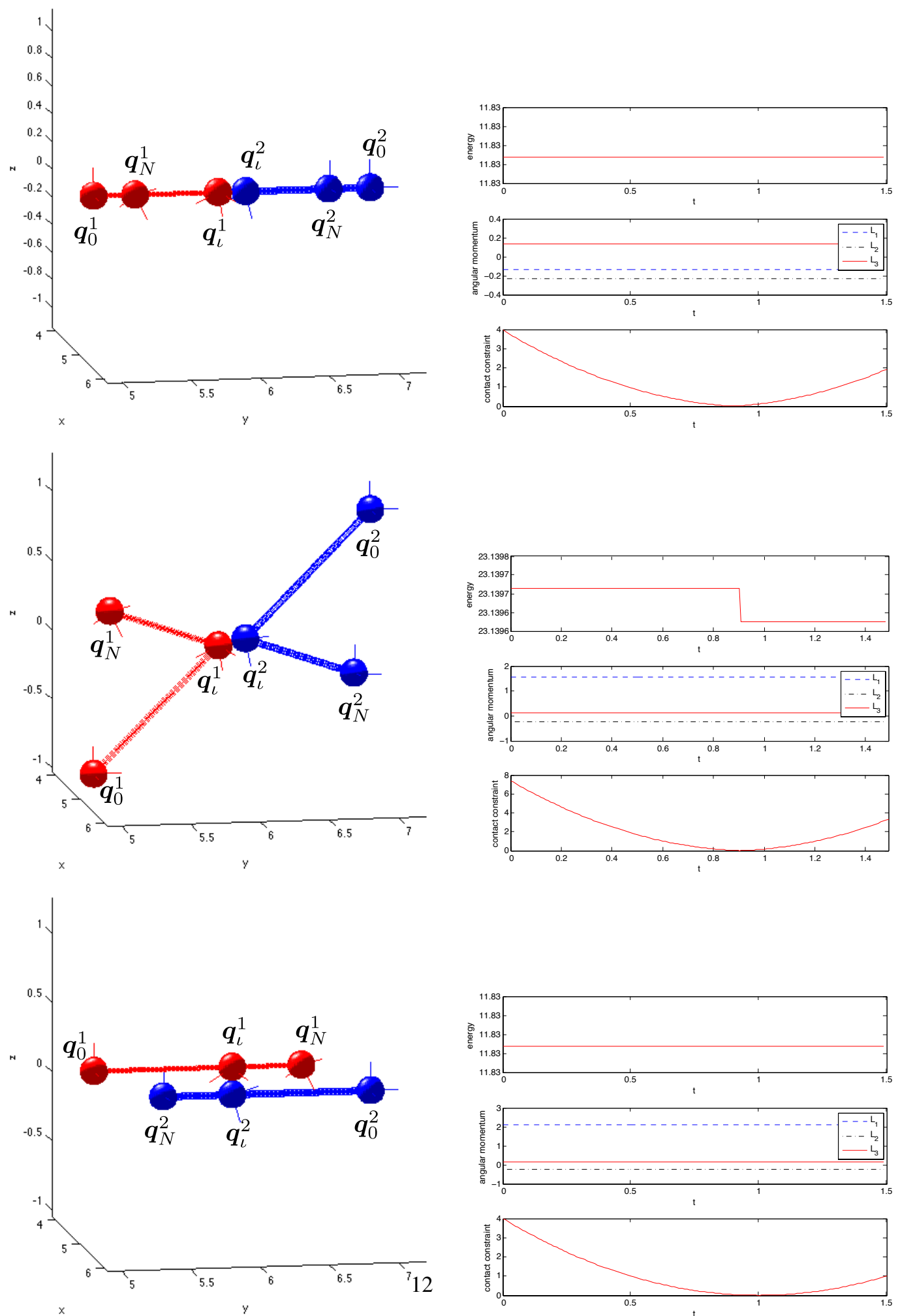

Figure 2: Trajectory and evolution of energy, angular momentum (conservation up to numerical tolerance) and contact constraint for two rigid spheres. 


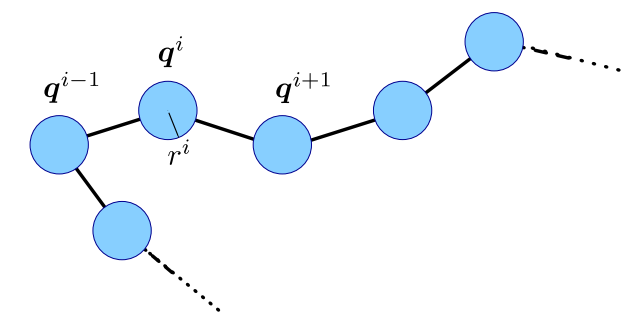

Figure 3: Model of a chain of beads.

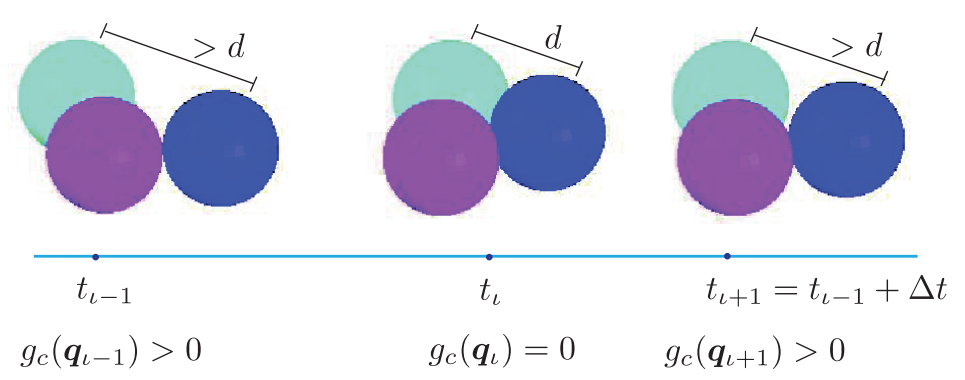

Figure 4: Pre-collision, collision and post-collision trimer configuration.

For the kinematic mass point chain, the discrete generalised coordinates

$\boldsymbol{u}_{n+1}=\left(\boldsymbol{u}_{n+1}^{1},\left(u_{r}^{2}\right)_{n+1},\left(u_{s}^{2}\right)_{n+1}, \ldots,\left(u_{r}^{n_{m p}}\right)_{n+1},\left(u_{s}^{n_{m p}}\right)_{n+1}\right)$ comprise the first mass' displacement and two incremental rotations for each subsequent mass. The discrete reparametrisation $\boldsymbol{q}_{n+1}=$ $\boldsymbol{F}_{d}\left(\boldsymbol{u}_{n+1}, \boldsymbol{q}_{n}\right)$ reads

$$
\begin{aligned}
& \boldsymbol{q}_{n+1}^{1}=\boldsymbol{q}_{n}^{1}+\boldsymbol{u}_{n+1}^{1} \\
& \boldsymbol{q}_{n+1}^{i}=\boldsymbol{q}_{n+1}^{i-1}+l^{i} \exp _{\boldsymbol{d}_{n}^{i}}\left(\left(u_{r}^{i}\right)_{n+1} \boldsymbol{r}_{n}^{i}+\left(u_{s}^{i}\right)_{n+1} \boldsymbol{s}_{n}^{i}\right)
\end{aligned}
$$

with the exponential map $\exp _{d}: T_{d} S^{2} \rightarrow S^{2}$ given by

$$
\exp _{d}(v)=\cos (\|v\|) d+\frac{\sin (\|v\|)}{\|v\|} v
$$

Remark 4 (Branched and looped polymers). Note that for simplicity of exposition, a linear kinematic chain is described here. However, the methodology can be easily applied to branched polymer chains and to molecules with loops. For details regarding the discrete null space reduction we refer to [3, 4, 25].

\subsection{Numerical example: intracolliding trimer}

This numerical example considers a trimer, thus $n_{m p}=3$, consisting of (orientationless) spheres of radius $r^{i}=\frac{d}{2}=0.5$ with mass $m^{i}=1$ for $i=1,2,3$ and initial distances between the masses $l^{2}=l^{3}=1$, see Figure 4. At time $t_{0}=0$, the masses are placed at $\boldsymbol{q}_{0}=$ $(1,0,0,0,0,0,0,-1,0)$ and the initial velocity, being consistent with the hidden constraints, is $\dot{\boldsymbol{q}}_{0}=(0,0.5,0,0,0,0,-0.5,0,0)$. No potential energy is present in this example. Except around 
collisions, time nodes are equispaced with $h=0.5$. The possibility to use a relatively large basic time-step shows the robustness of the variational integrator. Using a Newton-Raphson iteration, the discrete equations of motion have been solved up to a tolerance of $10^{-12}$. Figures 5 and 6 show results of the simulation of 10 and 10000 collisions using the variational integrator with the discrete null space reduction. The most important observation is the good energy behaviour which is typical for symplectic integration schemes. Even-though a large basic time-step is used and many collisions occur, the energy does not show any drift. It oscillates quasi-periodically with a small amplitude (order of magnitude $10^{-2}$ ) around the correct value during this longterm simulation. This amplitude (the energy error being the difference between upper and lower envelope) decreases quadratically for decreasing basic time-steps. The right panel of Figure 5 shows that during a normal time interval (without collision), typically $4-5$ Newton iterations are required, while $7-9$ iterations are necessary in time intervals with collisions. Always, the last configuration $\boldsymbol{q}_{n}$ is used as an initial guess for the iteration. Around collisions, the presence of the discrete energy conservation requires a more careful treatment: to ensure that iterates do not leave the radius of convergence, an ad-hoc damping strategy has been implemented which ensures that the Euclidian norm of iterative increments is not larger than the basic time-step, i.e. $\left\|\boldsymbol{u}_{n+1}^{l+1}\right\| \leq h$. The diagrams show that in some - but not in all - time intervals with collisions, one Newton increment has been damped.

Simulations using the variational integrator with the Lagrange multiplier method show indistinguishable results concerning the energy evolution. However, as the basic time-step gets smaller, slightly more Newton iterations are required while damping occurs a little less frequently. The most significant difference is that due to the presence of the Lagrange multipliers, the condition number becomes much larger as the basic time-step decreases compared to that in the discrete null space method (see Figure 7).

The comparison of the variational collision integrator with existing methods is summarised in Table 1. In [17], the same trimer example has been simulated using a collocation-based partitioned Gauss-Runge-Kutta method. Just like the variational integrator used here, this method is symplectic, momentum conserving and reversible. Furthermore, it preserves quadratic invariants. In the case of the trimer, energy fluctuations are in the range of $10^{-7}$, i.e., energy is preserved to higher accuracy than for the variational scheme where fluctuations are in the range of $10^{-2}$; see Figure 6. In the purely configuration based formulation of the discrete equations of motion in the variational integration scheme, the number of unknowns is generally only half as large as in configuration-velocity or configuration-momentum based formulations. While the Lagrange multiplier method increases the number of unknowns in the presence of constraints, their number is reduced to the minimum with the discrete null space reduction. In this example, the number of unknowns in our new approach is about 23 times smaller than in the configuration-velocity based collocation method with Lagrange multipliers and $s=10$ stages (which are necessary to cope with the large basic time-step of 0.5). In [17], the collision time is determined via a refinement strategy (with the same number of stages) between those collocation points where the contact takes place. The impulsive contribution of collisions is computed analytically. However, the large condition numbers in the range of $10^{12}$ caused by the presence of Lagrange multipliers limits the possible collision-time accuracy to $10^{-4}$. Between 4 and 5 refinements are necessary to determine the collision time up to this accuracy. In contrast to that, for each collision, we solve (15)-(16) to the aforementioned tolerance of $10^{-12}$. The larger number of unknowns together with the higher number of required time-steps in the collocation approach with refinement increases the computational costs by a factor of 13 compared to the variational scheme 

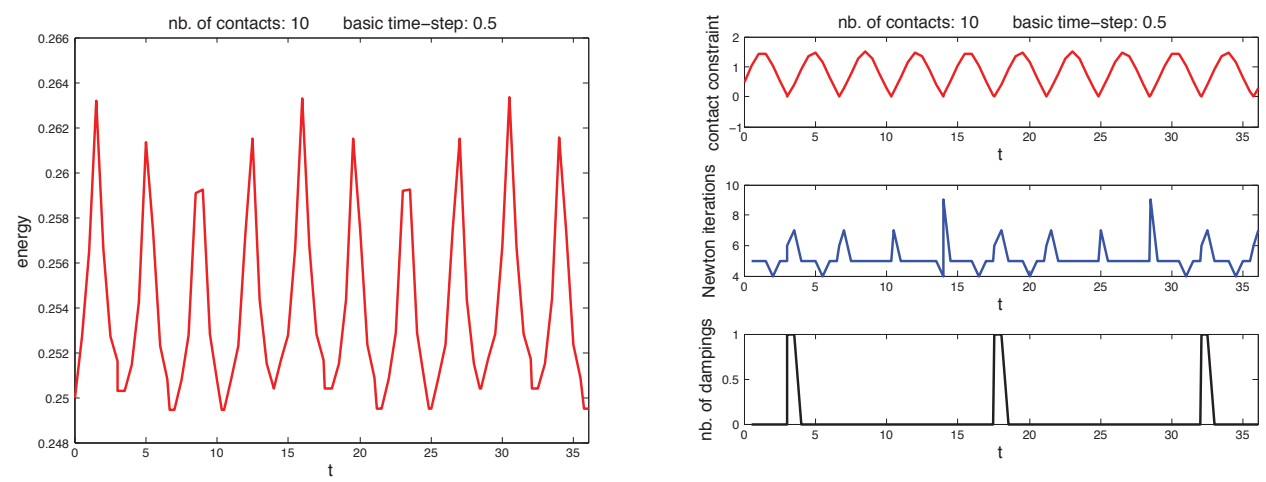

Figure 5: Intracolliding trimer: evolution of energy, inequality constraint, number of Newton iterations and number of necessary dampings during a simulation of 10 collisions using the variational integrator with the discrete null space reduction.

\begin{tabular}{l||c|c|c} 
& $\begin{array}{c}\text { variational integrator } \\
\text { null space reduction }\end{array}$ & $\begin{array}{c}\text { collocation } \\
\text { stage based refinement }\end{array}$ & $\begin{array}{c}\text { RATTLE } \\
\text { bisection }\end{array}$ \\
\hline \hline nb. unknowns & 65750 & 1540000 & 1251668 \\
\hline CPU-time & $<1 \mathrm{~min}$. & $13 \mathrm{~min}$. & $6.5 \mathrm{~min}$. \\
\hline max. condition & $10^{2}$ & $10^{12}$ & \\
\hline accuracy $t_{\iota}$ & $10^{-12}$ & $10^{-4}$ & $10^{-4}$ \\
\hline energy fluctuation & $10^{-2}$ & $10^{-7}$ & $10^{-3} \mathrm{drift}$
\end{tabular}

Table 1: Intracolliding trimer: comparison of the trimer motion with 1000 collisions simulated using the variational integrator with the discrete null space reduction, a collocation method with $s=10$ and RATTLE.

with discrete null space reduction. Both methods are comparable concerning the fulfilment of the constraints on configuration and velocity level and the conservation of angular momentum. Figure 8 shows an initial segment of the simulations using the variational integrator with discrete null space reduction and the collocation approach. As can be seen, the differing collision-time detection strategies cause a slight phase shift.

We moreover compared the variational scheme to a SHAKE/RATTLE integrator. The method becomes explicit when the Lagrange multipliers are solved for by successive linearisations employing a nonlinear one-step Gauss-Seidel iteration (see [22]). Using a basic time-step larger than 0.1 was not possible. Collision times have been determined via a classical bisection strategy and 6 bisections have been required to determine the collision time to an accuracy of $10^{-4}$. The number of unknowns is comparable to that of the collocation simulation, but the explicit RATTLE simulation is only half as expensive, and therefore it is still approximatey 6.5 times slower than the variational scheme with discrete null space reduction. Even-though after 1000 collisions the energy fluctuations are still relatively small, the energy shows an obvious drift (due to the still relatively large time-step). 

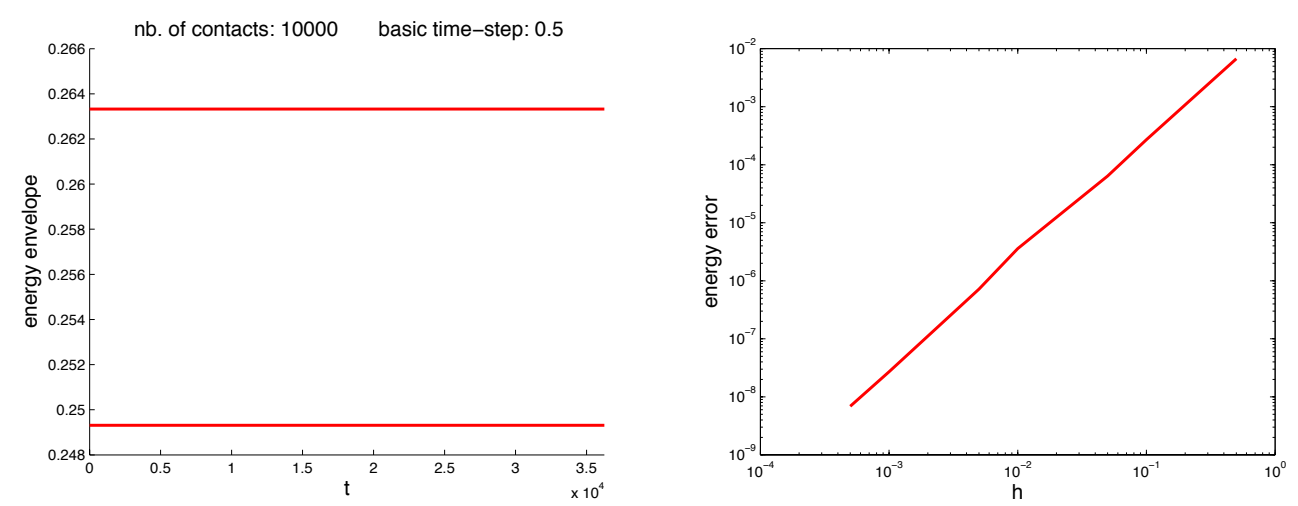

Figure 6: Intracolliding trimer: envelope of energy evolution during a simulation of 10000 collisions using the variational integrator with the discrete null space reduction. The energy oscillates quasi-periodically as in Figure 5 between the upper and lower envelope. The energy error (difference between upper and lower envelope) decreases quadratically for decreasing basic time-steps.

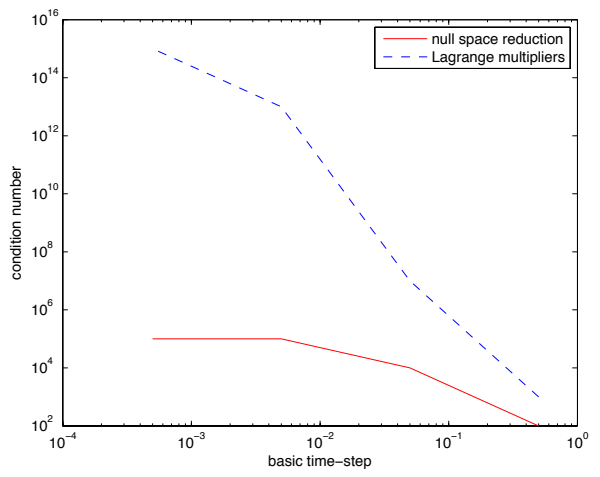

Figure 7: Intracolliding trimer: condition number of the iteration matrix for the variational integrator with Lagrange multipliers and with null space reduction. 

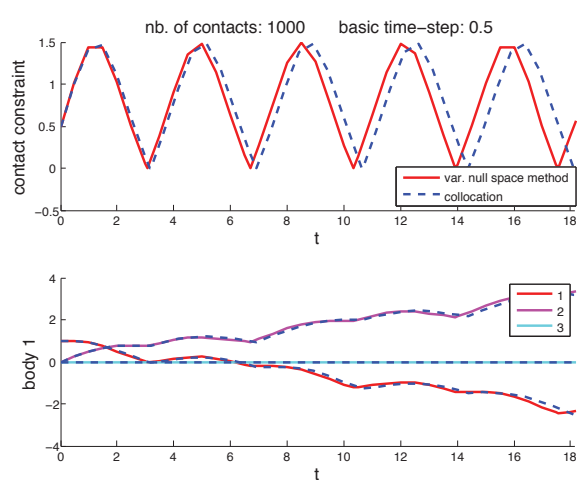

Figure 8: Intracolliding trimer: initial segment of the contact constraint and evolution of the first body's coordinates for the variational integrator and the collocation approach. 


\subsection{Numerical example: chain of four beads in a box}

As an example of a more complicated, non-integrable model, we consider a linear chain of four beads in a three-dimensional box. The trimer from Section 4.4 has been extended by one additional bead (of the same mass, length and radius) and placed in a box of size $8 \times 10 \times 14$ (relative to the bead size with radius $r=0.1$ ). Depending on the initial conditions, planar or real three-dimensional, periodic or non-periodic motion takes place where multiple collisions (between multiple beads or between multiple beads and multiple walls) occur.

Periodic motion with simultaneously happening contacts. The aim of the first simulation with initial conditions

$$
\begin{aligned}
& \boldsymbol{q}_{0}=\left(-\frac{3}{2 \sqrt{2}},-\frac{1}{2 \sqrt{2}}, 0,-\frac{1}{2 \sqrt{2}}, \frac{1}{2 \sqrt{2}}, 0, \frac{1}{2 \sqrt{2}},-\frac{1}{2 \sqrt{2}}, 0, \frac{3}{2 \sqrt{2}}, \frac{1}{2 \sqrt{2}}, 0\right) \\
& \dot{\boldsymbol{q}}_{0}=\left(\frac{1}{6}, \frac{1}{6}, 0, \frac{1}{6}, \frac{1}{6}, 0,-\frac{1}{6},-\frac{1}{6}, 0-\frac{1}{6},-\frac{1}{6}, 0\right)
\end{aligned}
$$

is to demonstrate the ability of the algorithm to handle simultaneously happening collisions in a stable way over a long range of time. The chain moves periodically between the collision configurations shown in Figure 9. Note that colliding beads are coloured in blue. Even after 10000 collisions (with 5000 corresponding collision time nodes), the collision times do not drift apart and the energy oscillates quasi-periodically with an amplitude of the order of magnitude $10^{-2}$ without any drift, see Figure 10.

Non-integrable three-dimensional motion. Initially, the chain is located as in the last example, however the initial velocity

$$
\dot{\boldsymbol{q}}_{0}=\left(-\frac{1}{10}, \frac{1}{10},-\frac{1}{10}, 0,0,0,0,0,0,0,0,0\right)
$$

leads to chaotic motion of the chain. Figure 11 illustrates different configurations, where beads in contact with other beads are coloured in blue while the red beads are in contact with a wall of the box. Note that the third row depicts a bead-bead collision happening a very short time before a bead-wall collision and the fourths row shows a bead-wall collision followed immediately by a bead-bead collision. In particular, the time between the described collisions is shorter than the basic time-step $h=0.1$. Obviously, the algorithm is capable to handle multiple collisions (at one time node) and collisions following each other rapidly in a stable way exhibiting good energy behaviour (fluctuations are are of the order of magnitude $10^{-4}$, they are not periodic as in the previous example, however we do not observe drift, see Figure 12).

\section{Conclusion}

Starting from a discrete version of Hamilton's principle, we have derived a structure preserving integrator for mechanical systems including holonomic (bilateral) constraints as well as unilateral contact (inequality) constraints. The basic idea of the discrete variational principle for systems involving collisions is to treat both the discrete-time trajectory and unknown collision time as unknowns; the resulting discrete Euler-Lagrange equations plus boundary conditions then lead to a time-stepping algorithm that includes the contact force and the constraint forces corresponding to the holonomic constraints. As we have shown all reaction forces can be eliminated 

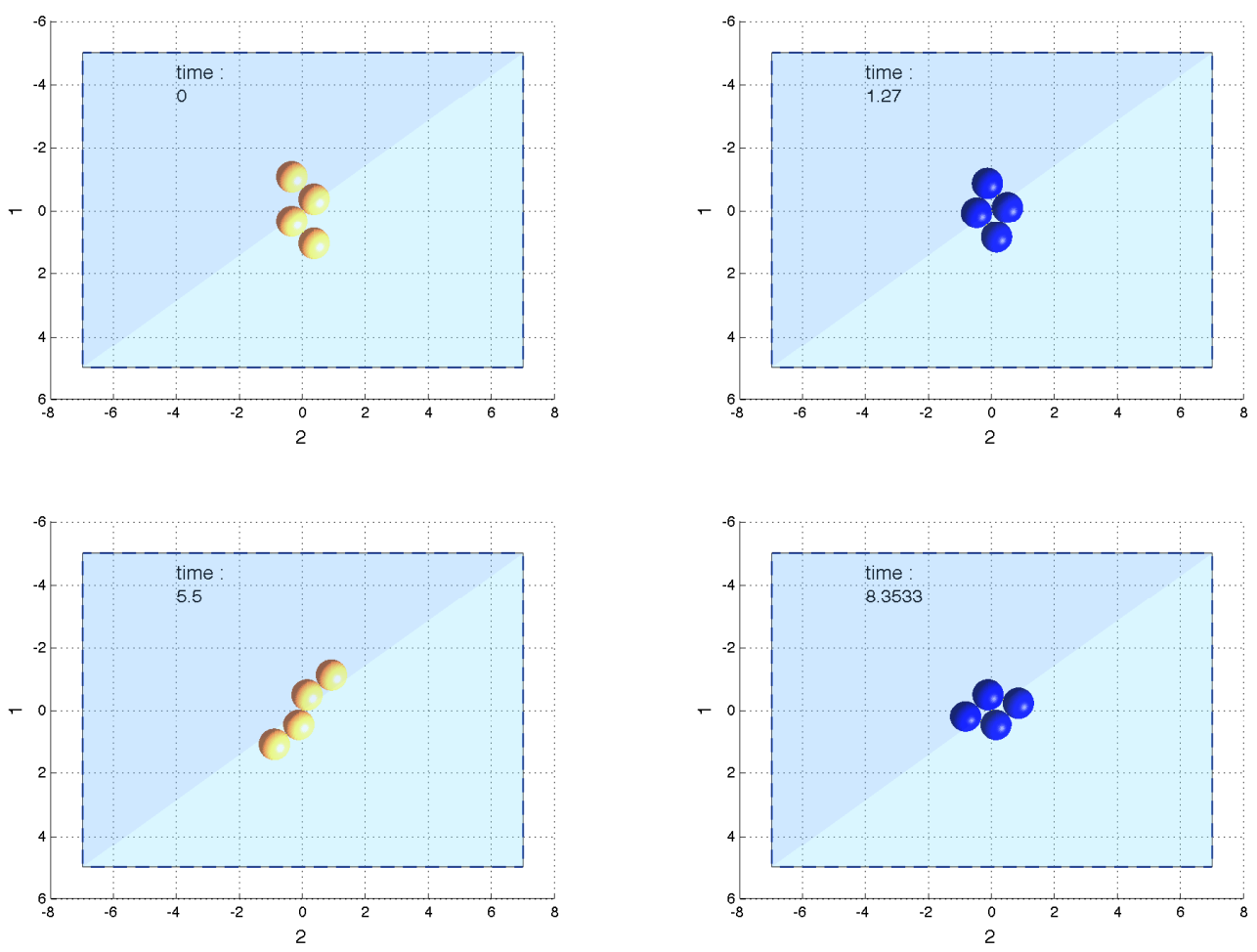

Figure 9: Chain of four beads, periodic motion: initial, intermediate and collision configurations.

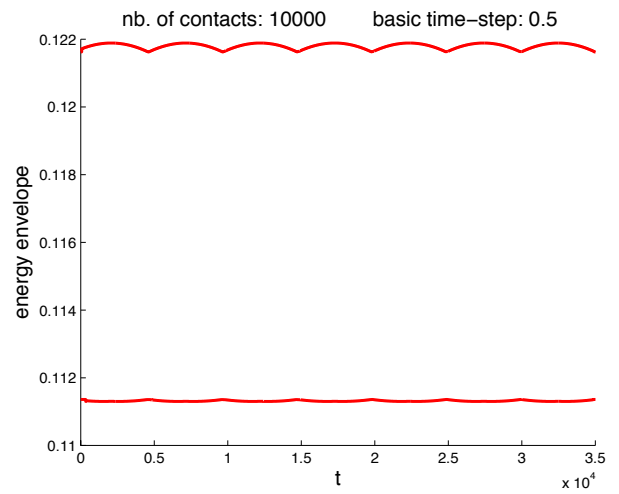

Figure 10: Chain of four beads, planar periodic motion: envelope of energy evolution during a simulation of 10000 collisions (at 5000 time nodes, two collisions take place simultaneously). 

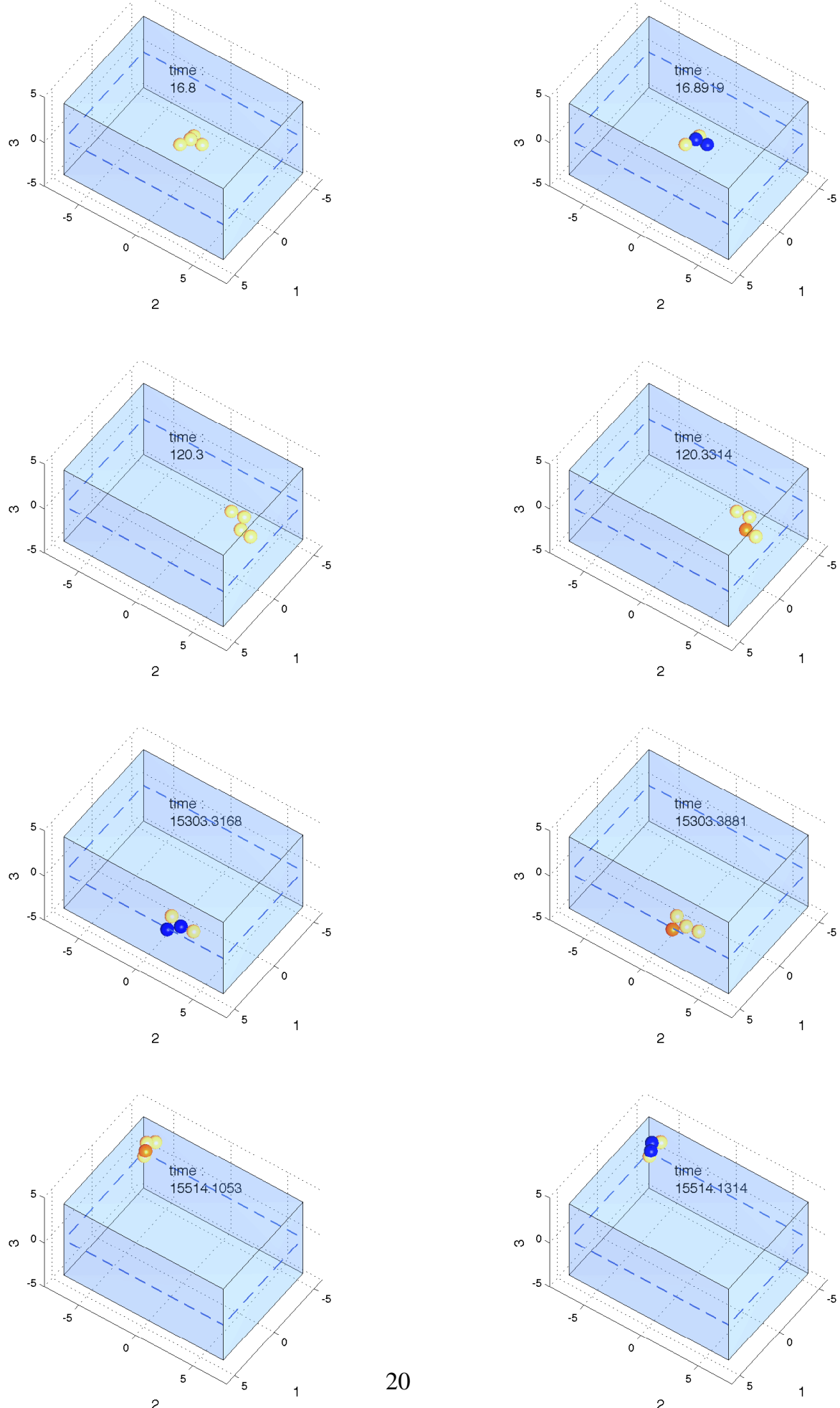

Figure 11: Chain of four beads, chaotic motion: different configurations including bead-bead and bead-wall collisions following each other rapidly. 

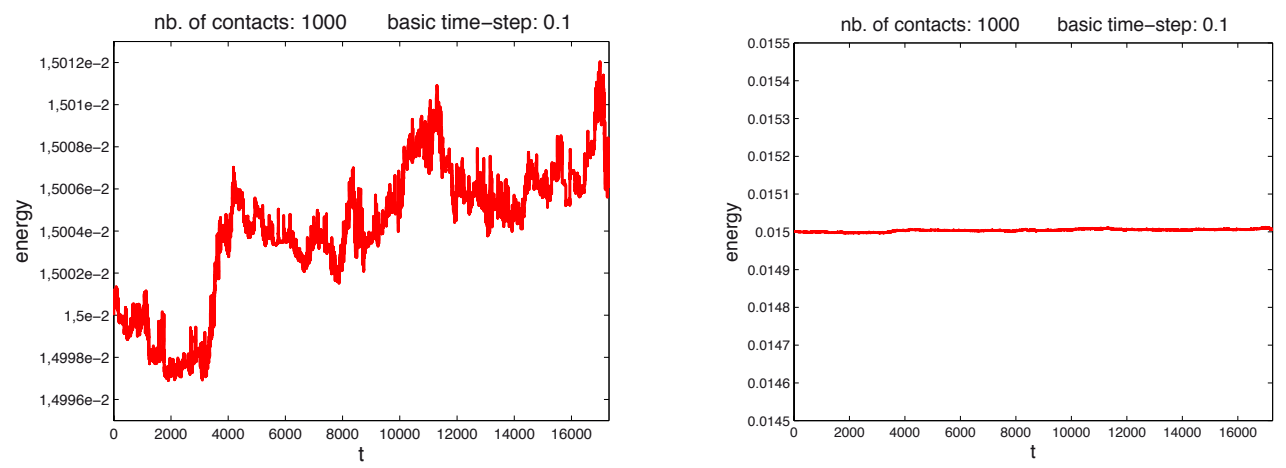

Figure 12: Chain of four beads, chaotic motion: evolution of energy during a simulation of 1000 collisions, including various collisions following each other in quick succession.

from the discrete equations of motion using a discrete null space reduction (involving a projection and a local reparametrisation step) which considerably reduces the number of unknowns as compared to the standard treatment with Lagrange multipliers. As a consequence, the condition number during iteration stays low. This fact, together with the possibility to determine the contact time by solving an algebraic equation rather than searching it via bisection strategies, leads to much lower computational costs while, at the same time, it increases the accuracy to which the contact time is determined. Although the overall scheme is not symplectic in any obvious sense, we found that it shows excellent long-term stability and quasi-energy-conservation akin to symplectic integrators: the total energy remains close to the correct value, even after many thousands of collisions using a large basic time-step, and even in case of multiple collisions happening at the same time node or following each other in quick succession. We stress that the method is rather flexible and can (and should) be extended to, e.g., non-spherical particle geometries or systems involving a smooth interaction potential. For large particle systems, in which the particle density varies in different regions, i.e., collisions occur more frequently in some parts of the system than in others, a combination with a multiple time-stepping strategy (a multirate integrator $[15,16]$ or an asynchronous integrator [24]) might be worthwhile. A still open problem is the backward error analysis of non-smooth systems with variable time-step that will be addressed in future work.

\section{References}

[1] Alder, B., Wainwright, T., 1959. Studies in molecular dynamics. i. general method. J. Chem. Phys. 31, 459-466.

[2] Andersen, H., 1983. Rattle: A velocity version of the shake algorithm for molecular dynamics calculations. J. Comput. Physics 52, 24-34.

[3] Betsch, P., 2005. The discrete null space method for the energy consistent integration of constrained mechanical systems. Part I: Holonomic constraints. Comput. Methods Appl. Mech. Engrg. 194 (50-52), 5159-5190.

[4] Betsch, P., Leyendecker, S., 2006. The discrete null space method for the energy consistent integration of constrained mechanical systems. Part II: Multibody dynamics. Int. J. Numer. Meth. Engng. 67 (4), $499-552$.

[5] Binder, K., 1995. Monte Carlo and Molecular Dynamics Simulations in Polymer Science. Oxford University Press.

[6] Bond, S., Leimkuhler, B., 2007. Molecular dynamics and the accuracy of numerically computed averages. Acta Numerica 16, 1-65.

[7] Bond, S., Leimkuhler, B., 2007. Stabilized integration of hamiltonian systems with hard-sphere inequality constraints. SIAM Journal on Scientific Computing 30 (1), 134-147.

[8] Bridges, T., Reich, S., 2006. Numerical methods for Hamiltonian PDEs. J. Phys. A: Math. Gen. 39, 5287-5320. 
[9] Cirak, F., West, M., 2005. Decomposition contact response (dcr) for explicit finite element dynamics. Int. J. Numer. Meth. Engng. 64, 1078-1110.

[10] de la Pena, L., van Zon, R., Schofield, J., Opps, S., 2007. Discontinuous molecular dynamics for semiflexible and rigid bodies. J. Chem. Phys. 126 (074105).

[11] Doi, M., Edwards, S., 1986. The Theory of Polymer Dynamics. Clarendon Press, Oxford.

[12] Dontchev, A., Lempio, F., 1992. Diffference methods for differential inclusions: A survey. SIAM Rev. 34, $263-284$.

[13] Fetecau, R., Marsden, J., Ortiz, M., West, M., 2003. Nonsmooth lagrangian mechanics and variational collision integrators. Siam J. applied dynamical systems 2 (3), 381-416.

[14] Frenkel, D., Maguire, J., 1983. Molecular dynamics study of the dynamical properties of an assembly of infinitely thin hard rods. Mol. Phys. 49, 503-541.

[15] Gear, C., Wells, R., 1984. Multirate linear multistep methods. BIT 24, 484502.

[16] Hairer, E., Wanner, G., Lubich, C., 2006. Geometric Numerical Integration: Structure-Preserving Algorithms for Ordinary Differential Equations. Springer.

[17] Hartmann, C., Schütte, C., Kalibaeva, G., Di Pierro, M., Cicotti, G., 2009. Fast simulation of polymer chains. J. Chem. Phys. 130.

[18] Houndonougbo, Y., Laird, B., Leimkuhler, B., 2000. A molecular dynamics algorithm for mixed hardcore/continuous potentials. Mol. Phys. 98, 309-316.

[19] Jay, L., 2007. Beyond conventional Runge-Kutta methods in numerical integration of ODEs and DAEs by use of structures and local models. J. Comput. Appl. Math. 204, 56-76.

[20] Jay, L., 2007. Specialized partitioned additive Runge-Kutta methods for systems of overdetermined DAEs with holonomic constraints. SIAM J. Numer. Anal. 45, 1814-1842.

[21] Leimkuhler, B., Reich, S., 1994. Symplectic integration of constrained Hamiltonian systems. Mathematics of Computations 63, 589-605.

[22] Leimkuhler, B., Reich, S., 2004. Simulating Hamiltonian Dynamics. Cambridge University Press

[23] Lempio, F., Veliov, V., 1998. Discrete approximations of differential inclusions. Bayreuth. Math. Schr. 54, 149-232.

[24] Lew, A., Marsden, J., Ortiz, M., West, M., 2003. Asynchronous variational integrators. Arch. Rational Mech. Anal. $167,85-146$

[25] Leyendecker, S., Marsden, J., Ortiz, M., 2008. Variational integrators for constrained dynamical systems. ZAMM 88 (9), 677-708.

[26] Marsden, J., West, M., 2001. Discrete mechanics and variational integrators. Acta Numerica 10, 357-514.

[27] McNeil, W., Madden, W., 1982. A new method for the molecular dynamics simulation of hard core molecules. The Journal of Chemical Physics 76, 6221-6226.

[28] Padding, J., Briels, W., 2011. Systematic coarse-graining of the dynamics of entangled polymer melts: the road from chemistry to rheology. J. Phys.: Condens. Matter 23 (23), 233101.

[29] Rapaport, D., 1979. Molecular dynamics study of a polymer chain in solution. J. Chem. Phys. 71, 3299-3303.

[30] Reich, S., 1999. Backward error analysis for numerical integrators. SIAM J. Numer. Anal. 36, 1549-1570.

[31] Ryckaert, J., Ciccotti, G., Berendsen, H., 1977. Numerical integration of the Cartesian equations of motion of a system with constraints: Molecular dynamics of n-alkanes. J. Comput. Phys. 23, 327-341.

[32] Stratt, R., Holmgren, S., Chandler, D., 1981. Constrained impulsive molecular dynamics. Mol. Phys. 42 (12331243).

[33] Suh, S., Mier-y Teran, L., White, H., Davis, H., 1990. Molecular dynamics study of the primitive model of 1-3 electrolyte solutions. Chem. Physics 142, 203-211.

[34] Wendlandt, J., Marsden, J., 1997. Mechanical integrators derived from a discrete variational principle. Physica D 106, 223-246

[35] Zeidler, E., 1995. Applied Functional Analysis: Main Principles and Their Applications (Applied Mathematical Sciences, Vol. 109). Springer, Berlin.

[36] Zhou, Y., Karplus, M., 1999. Folding of a model three-helix bundle protein: a thermodynamic and kinetic analysis. Journal of Molecular Biology 293 (4), 917 - 951. 